\title{
Síntesis y caracterización de un compuesto semiconductor NiO-ZnO dopado con nanopartículas de Au por el método sol-gel para aplicación como sensores de gas
}

\author{
Alex Díaz ${ }^{1}$, Dionicio Otiniano ${ }^{1}$, E. Della Gaspera ${ }^{2}$, Alessandro Martucci $^{2}$ \\ 1. Departamento de Ingeniería de Materiales, Universidad Nacional de Trujillo-Perú \\ ${ }^{2}$ Dipartimento d'Ingegneria Meccanica - Settore Materiali, Universita di Padova, 35131 Padova-Italia
}

\section{RESUMEN}

Láminas porosas de un compuesto semiconductor formado por NiO-ZnO (\%mol) dopado con nanopartículas de $\mathrm{Au}(3 \% \mathrm{~mol})$ fueron preparados por el método sol-gel usando acetato de níquel tetrahidratado $\left(\mathrm{NiC}_{4} \mathrm{H}_{6} \mathrm{O}_{4} .4 \mathrm{H}_{2} \mathrm{O}\right)$ y acetato de zinc dihidratado $\left(\mathrm{C}_{4} \mathrm{H}_{6} \mathrm{O}_{4} \mathrm{Zn} .2 \mathrm{H}_{2} \mathrm{O}\right)$ como precursores, metanol $\left(\mathrm{CH}_{6} \mathrm{OH}\right)$ y etanol $\left(\mathrm{C}_{2} \mathrm{H}_{6} \mathrm{O}\right)$ como solventes, monoetanolamina $\left(\mathrm{C}_{2} \mathrm{H}_{7} \mathrm{NO}\right)$ y dietanolamina $\left(\mathrm{C}_{4} \mathrm{H}_{11} \mathrm{NO}_{2}\right)$ como ligantes funcionales, y ácido cloroaúrico $\mathrm{HAuCl}_{4}$ como precursor. Las muestras se caracterizaron por espectroscopias Infrarrojo (IR), ultravioleta (UV-VIS), microscopía SEM, difracción de rayos X (XRD), y ensayos de sensores gaseosos. Las muestras semiconductoras fueron depositadas sobre substratos de silicio por el método de spin-coating a $2000 \mathrm{rpm}$, posteriormente fueron tratadas a 500 y $600^{\circ} \mathrm{C}$. Los efectos de las composiciones de $\mathrm{NiO}-\mathrm{ZnO}$ y el porcentaje de dopaje también se discuten en este argumento. El espesor de la capa fue determinado por elipsometria aproximado a $75 \mathrm{~nm}$. Estos compuestos fueron ensayados para sensores gaseosos de $\mathrm{H}_{2}$ y CO $(1 \% \mathrm{~V} / \mathrm{V})$ a $300^{\circ} \mathrm{C}$, demostrando óptimos resultados para el $\mathrm{H}_{2}$, pero no así para el $\mathrm{CO}$. Descriptores: NiO, $\mathrm{ZnO}$, semiconductores, sensor gaseoso.

\section{ABSTRACT}

Porous films formed by a semiconductor $\mathrm{ZnO}-\mathrm{NiO}$ (\% mol) doped with $\mathrm{Au}$ nanoparticles ( $3 \%$ mol) were prepared by sol-gel method using nickel acetate tetrahydrate $\left(\mathrm{NiC}_{4} \mathrm{H}_{6} \mathrm{O}_{4} .4 \mathrm{H}_{2} \mathrm{O}\right)$ and zinc acetate dihydrate $\left(\mathrm{C}_{4} \mathrm{H}_{6} \mathrm{O}_{4} \mathrm{Zn} .2 \mathrm{H}_{2} \mathrm{O}\right)$ as precursors, methanol $\left(\mathrm{CH}_{6} \mathrm{OH}\right)$ and ethanol $\left(\mathrm{C}_{2} \mathrm{H}_{6} \mathrm{O}\right)$ as solvents, monoethanolamine $\left(\mathrm{C}_{2} \mathrm{H}_{7} \mathrm{NO}\right)$ and diethanolamine $\left(\mathrm{C}_{4} \mathrm{H}_{11} \mathrm{NO}_{2}\right)$ as functional chelants, and chloroauric acid $\left(\mathrm{HAuCl}_{4}\right)$ as gold precursor. The samples were characterized by infrared (IR) and ultraviolet (UV-VIS) spectroscopy, microscopy SEM, X-ray diffraction (XRD) and gas sensing tests. The semiconductor samples were deposited on silicon substrates by spin-coating method at $2000 \mathrm{rpm}$, subsequently annealing at 500 and $600{ }^{\circ} \mathrm{C}$. The effects of the compositions of $\mathrm{NiO}-\mathrm{ZnO}$ and the percentage of doping are also discussed. The layer thickness was determined by ellipsometry in approximately $75 \mathrm{~nm}$. These compounds were tested for gas sensors for $\mathrm{H}_{2}$ and $\mathrm{CO}(1 \% \mathrm{~V} / \mathrm{V})$ at $300^{\circ} \mathrm{C}$, showing excellent results for $\mathrm{H}_{2}$, but not for the $\mathrm{CO}$.

Keywords: $\mathrm{NiO}, \mathrm{ZnO}$, semiconductors, gas sensor. 


\section{INTRODUCCION}

La capacidad humana de diferenciar entre cientos de olores está bien desarrollada; pero falla si se deben detectar concentraciones absolutas de gas o gases inodoros [1]. Los sensores gaseosos se utilizan en aplicaciones de seguridad donde hay gases tóxicos 0 combustibles, y también en las denominadas aplicaciones de confort, tales como en el control de climas de edificios y vehículos donde se requiere una buena calidad del aire [2]. EI control de procesos industriales es otra aplicación de los sensores gaseosos junto con los análisis de laboratorio. Es por ello, que es necesario diseñar sensores gaseosos pequeños y baratos que posean alta sensibilidad, selectividad y estabilidad respecto a la aplicación de uso [3]. En la actualidad existe una gran variedad de sensores basados en diferentes propiedades como por ejemplo; sensores gaseosos semiconductores, sensores ópticos, sensores de conductividad térmica, sensores catalíticos, sensores dieléctricos, sensores electroquímicos y sensores electrolíticos [4].

El $\mathrm{ZnO}$ es un semiconductor tipo $\mathrm{n}$ con propiedades únicas tales como transparencia a la reflectividad infrarrojo, características acústicas, alta estabilidad electroquímica y excelente propiedades electrónicas. Ha sido ampliamente usado como sensor químico [5], instrumento superficial de onda acústica [6], y películas foto anódicas para celdas solares [7]. Una diversa variedad de técnicas para de la preparación de películas de $\mathrm{ZnO}$ se utilizan como: sputtering [8], deposición por vapor químico (CVD) [9], pirolisis spray [10], y procesos sol-gel [11-13].

$\mathrm{El} \mathrm{NiO}$ es uno de los pocos óxidos metálicos que es del tipo $p$, ha sido extensivamente estudiado debido a sus propiedades mecánicas, eléctricas, magnéticas y ópticas. Las nanopartículas de $\mathrm{NiO}$ son muy usadas en diferentes campos como catálisis [14], películas electrocrómicas [15], y electrodos de celdas para combustibles [16]. Varias técnicas se desarrollaron para la preparación del $\mathrm{NiO}$, entre ellas destacan: electro spinning [17], procesos de solventes orgánicos [18], y procesos sol-gel [19].

\section{METODOS}

\subsection{Síntesis}

Para las muestras de $\mathrm{ZnO}, 200 \mathrm{mg}$ de acetato de zinc dihidratado $(\mathrm{ZnAc})$ se disolvieron en 1.8 $\mathrm{mL}$ de etanol, y subsecuentemente se agregaron $0.066 \mathrm{~mL}$ de monoetanolamina (MEA) en agitación continua durante 45 minutos. Para el NiO, $300 \mathrm{mg}$ de acetato de niquel tetrahidratado (NiAc) se disolvieron en 2 $\mathrm{mL}$ de metanol y se agrego $0.18 \mathrm{~mL}$ de dietanolamina (DEA) en agitación durante 1 hora. Las nanopartículas de Au se sintetizaron disolviendo $18 \mathrm{mg}$ de $\mathrm{HAuCl}_{4}$ en $3 \mathrm{~mL}$ de metanol, se agrego a esta mezcla una solución metanolica de PVP-10K ( $75 \mathrm{mg}$ en $38 \mathrm{~mL}$ ) bajo agitación constante. Después de 30 minutos una solución preparada de $8 \mathrm{mg}$ de $\mathrm{NaBH}_{4}$ disuelto en $3 \mathrm{~mL}$ de metanol se agrego bajo fuerte agitación, y la solución resultante se dejo mezclar durante 30 minutos. Posteriormente el etanol es removido en un tambor de evaporación, y el sedimento es redispersado en agua. Las partículas son precipitadas con exceso de acetona, centrifugadas a $4000 \mathrm{rpm}$ durante $5 \mathrm{~min}$, y redispersado en etanol obteniendo una solución concentrada a $30 \mathrm{mM}$. Se determinaron las cantidades molares, y se hizo la mezcla correspondiente a $50 \mathrm{NiO}-50 \mathrm{ZnO}$ y 75NiO-25NiO. Los dopajes con las nanopartículas de $\mathrm{Au}$ se hicieron 10 minutos antes de efectuar la deposición sobre silicio.

\subsection{Preparación del filme y procesamiento}

Después del envejecimiento del sol, las muestras fueron depositadas sobre substratos de silicio usando un spin coater $\mathrm{KW}-4 \mathrm{~A} / \mathrm{Little}$ (Chemat Scientific) a una velocidad de rotación de $2000 \mathrm{rpm}$ en gas argón durante 40 segundos. Los substratos recubiertos fueron transferidos a un horno (Carbolite GSM 11/8) donde se mantuvieron a una temperatura de $300^{\circ} \mathrm{C}$. Después de 10 minutos el horno fue llevado a las temperaturas de 500 y $600{ }^{\circ} \mathrm{C}$ respectivamente a una velocidad aproximada de calentamiento de $10^{\circ} \mathrm{C} \cdot \mathrm{min}^{-1}$.

La espectroscopia de absorción infrarrojo, en el rango de 400 a $2500 \mathrm{~cm}^{-1}$, fue registrada en un espectroscopio Fourier Transform Infrared (FTIR)-JASCO serie 620. Para medir el espectro de absorción se uso un espectrofotómetro UV-VIS JASCO serie 570. Las muestras fueron caracterizadas por difracción de rayos $X$ (XRD) usando un difractómetro Philips PW1820 equipado con ángulo de incidencia óptica de rayos $\mathrm{X}$, el análisis se desarrollo a $0.5^{\circ}$ usando filtro de 
radiación $\mathrm{Cu} \mathrm{K} \alpha \mathrm{Ni}$ a $40 \mathrm{kV}$ y $40 \mathrm{~mA}$. La morfología de las películas se observaron usando un microscopio electrónico de barrido Philips XL-30 (ESEM). La respuesta eléctrica de las películas a los gases $\mathrm{H}_{2}$ y $\mathrm{CO}$ fueron medidos en un sistema automatizado. La funcionalidad del sensor óptico se determino con mediciones de absorbancia óptica entre el rango de longitud de onda de $300<\lambda<900$ con muestras montadas sobre un calentador en una celda de flujo

gaseoso especialmente diseñada. El tamaño de las muestras fue de aproximadamente de $1 \mathrm{~cm} \times 2 \mathrm{~cm}$, y el haz incidente del espectrofotómetro fue normal a la superficie de la muestra y cubre un área superficial de $6 \mathrm{~mm}$ $\times 1.5 \mathrm{~mm}$.

\section{RESULTADOS Y DISCUSION}

El análisis FTIR de las películas $75 \mathrm{NiO}-25 \mathrm{NiO}-$ $3 \% \mathrm{Au}$ depositados sobre silicio y tratadas a diferentes temperaturas se reportan en la Fig. 1. En estos espectros es evidente los picos dominantes del enlace Si-O alrededor de 1050 y $1150 \mathrm{~cm}^{-1}$, lo cual se relaciona con la porosidad total del filme. La posición de los picos Si-O aumentan con el incremento de la temperatura de tratamiento.

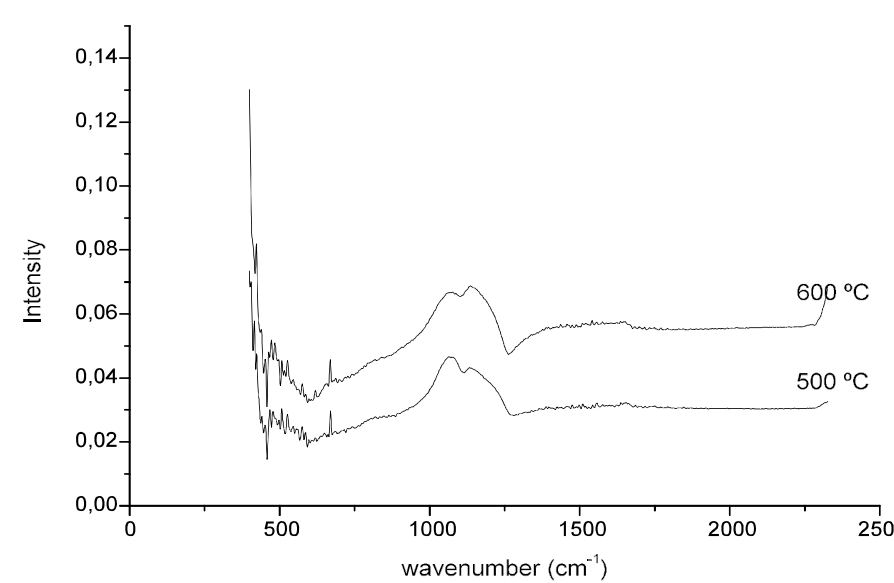

Figura 1: Espectro FTIR de un filme $75 \mathrm{NiO}-$ 25ZnO-3Au tratados a diferentes temperaturas.

En la figura 2, la medición de absorción óptica en el rango UV muestra en el compuesto al $50 \% \mathrm{NiO}$ el borde típico de absorción del $\mathrm{ZnO}$ cerca de $375 \mathrm{~nm}$. Los compuestos también muestran la banda de resonancia superficial plasmónica (SPR) de las nanopartículas de Au entre $570-630 \mathrm{~nm}$, siendo ligeramente superior y de mayor amplitud con el incremento del contenido de $\mathrm{NiO}$. Esto se puede explicar considerando los diferentes índices refractivos de los dos óxidos: para el $\mathrm{ZnO}$ de 2.01 [20], mientras que para el $\mathrm{NiO}$ de 2.33 [21], tal que si hay un incremento del $\mathrm{NiO}$ en el compuesto, resulta un incremento del promedio del índice refractivo y esto causa el aumento del pico observado.

La amplitud de la banda de Au puede deberse a la interacción del metal noble Au con el metal activo superficial $\mathrm{NiO}$, de hecho se ha demostrado que $\mathrm{Au}$ y $\mathrm{NiO}$ tienen la misma estructura cristalina cubica y pueden formar estructuras dobles en el cual los cristales se acoplan a lo largo de los planos $\mathrm{Au}(111)$ y $\mathrm{NiO}$ (200).

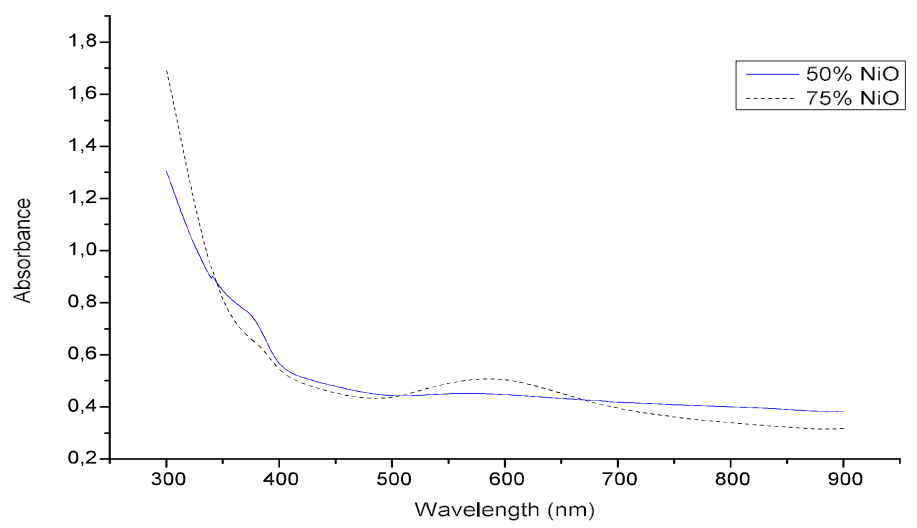

Figura 2. Espectro de absorción óptica para el compuesto ZnO-NiO dopado con nanopartículas de Au recocido a $600^{\circ} \mathrm{C}$.
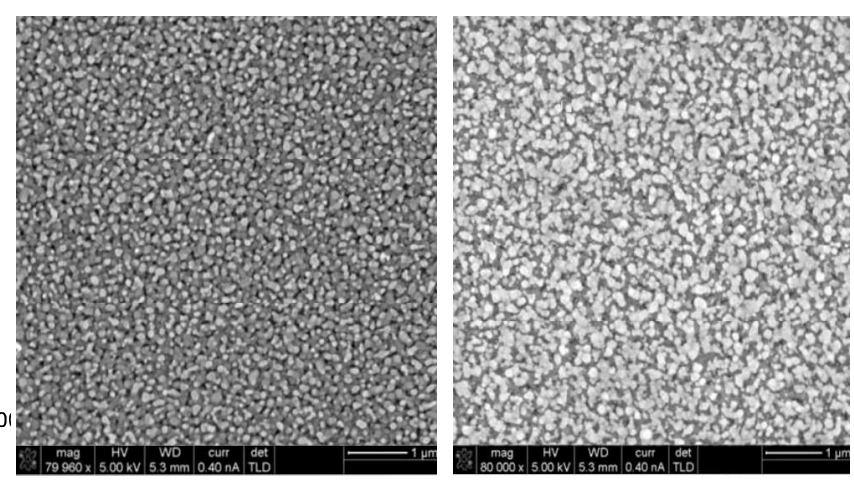

Figure 3.Microscopia electrónica de barrido (SEM) a) $\mathrm{ZnO}$ y b) compuesto $\mathrm{ZnO}-\mathrm{Au}$ recocido a $600{ }^{\circ} \mathrm{C}$.

Las microfotografías SEM muestran la morfología de la película que presentan los nanocompuestos en la figura 3 . Son fácilmente detectables los granos cristalinos del óxido de zinc, así como la presencia de una porosidad residual. La morfología observada es deseable para las películas delgadas de sensores de 
gas, con el fin de obtener un sensor con alta área superficial específica, y tener un camino fácil para que los gases entren y salgan de los nano-compuestos. Además se observa un filme compacto y sin presencia de fisuras a pesar de la alta temperatura de tratamiento.

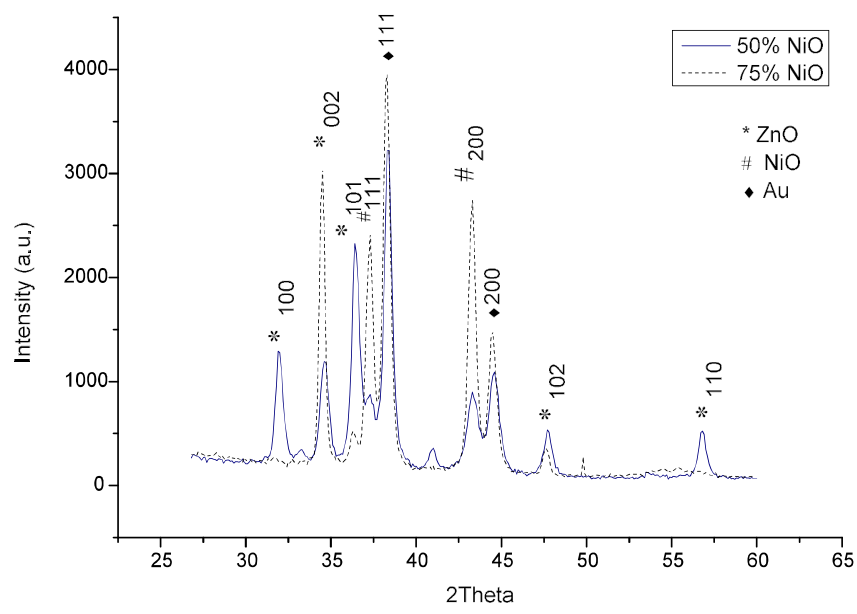

Figure 4. Difracción de rayos $X(X R D)$ de láminas compuestas $\mathrm{ZnO}-\mathrm{NiO}$ dopadas con nanopartículas de $\mathrm{Au}$ a $600^{\circ} \mathrm{C}$.

La difracción de rayos $X$ muestra los compuestos conteniendo $3 \%$ molar de $\mathrm{Au}$ a diferentes concentraciones de $\mathrm{ZnO}$ recocidos a $600{ }^{\circ} \mathrm{C}$ en la figura 4 . Todos los compuestos presentan fases cristalinas, debido a que la temperatura de $600^{\circ} \mathrm{C}$ es suficientemente alta para la pirolisis de los orgánicos dentro de la película, promoviendo la reducción de iones $A u$ disperso dentro de los óxidos, y también permite cristalizar ambos óxidos de $\mathrm{Ni}$ y $\mathrm{Zn}$. Analizando las reflexiones del $\mathrm{ZnO}$, el pico de difracción (002) es mucho más intenso que los demás, mientras que los otros picos son muy débiles, indicando una extensiva orientación a lo largo de los ejes c. Así también se nota claramente que los picos incrementan progresivamente en intensidad conforme aumenta el contenido del $\mathrm{Ni}$, indicando claramente una alta cantidad de $\mathrm{NiO}$ en el compuesto como se esperaba. Los picos de difracción para cada fase son muy similares en intensidad y amplitud. Aparte del incremento en la intensidad de los picos del $\mathrm{NiO}$, lo que confirma una estructura similar de los nanocompuestos. El tamaño promedio de los cristales pudo estimarse por la ecuación de Debye-Scherrer (ver ec. 1). En esta ecuación $\tau$ es el tamaño promedio del cristal, $\mathrm{K}$ es el factor forma de la ecuación ( 0.9 para esferas), $\lambda$ es la longitud de onda de los rayos $X(1.54 \AA), \beta$ es la línea del ancho total a la mitad de las máxima reflexiones (FWHM) y $\theta$ es el ángulo de Bragg. Siendo el tamaño de los cristales de $\mathrm{ZnO}, \mathrm{NiO}$ y $\mathrm{Au}$ entre 23-30 nm, 12-16 nm y 14-19 nm respectivamente en todas las muestras.

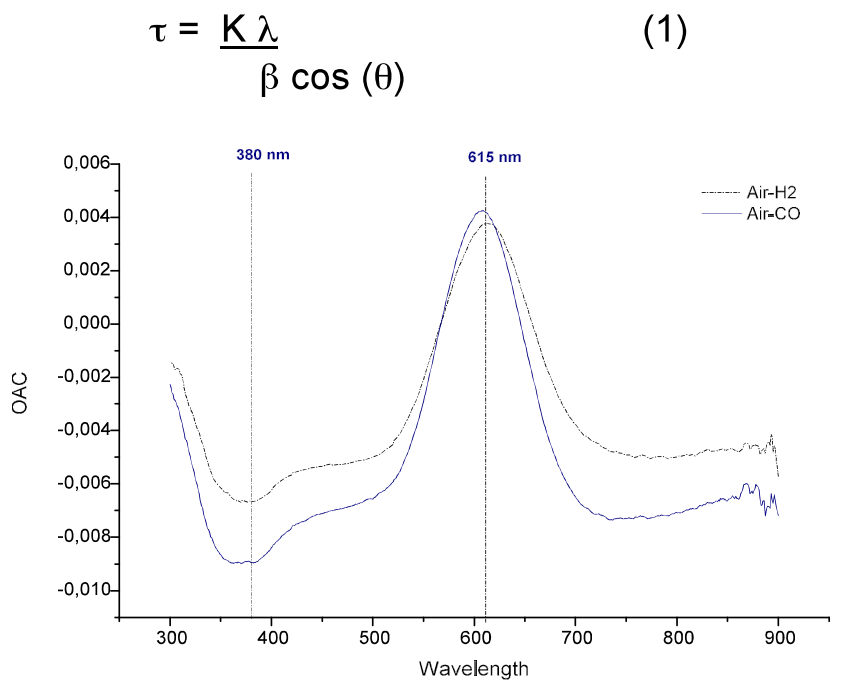

Figure 5. Curva $O A C$ en función de la longitud de onda para el $\mathrm{H}_{2}$ y $\mathrm{CO}$ a $300^{\circ} \mathrm{C}$.

Los ensayos de sensores gaseosos se desarrollaron a la temperatura de $300^{\circ} \mathrm{C}$, con una aceptable respuesta. Debajo de esta temperatura la respuesta de los sensores fue muy pequeña. La figura 5 reporta la curva de cambios de absorbancia óptica (OAC) ensayada para determinar la longitud optima para el ensayo, mostrando una respuesta simétrica con picos máximos positivos y de aproximadamente la misma intensidad. Los gases introducidos dentro de la cámara fueron $\mathrm{H}_{2}$ y $\mathrm{CO}(1 \% \mathrm{~V} / \mathrm{V})$ respectivamente. La absorbancia de estas láminas disminuye cuando se expone a los gases ensayados, como se esperaba debido a interacción entre gases reductores como $\mathrm{CO}$ y semiconductores tipo $\mathrm{p}$ como NiO. 


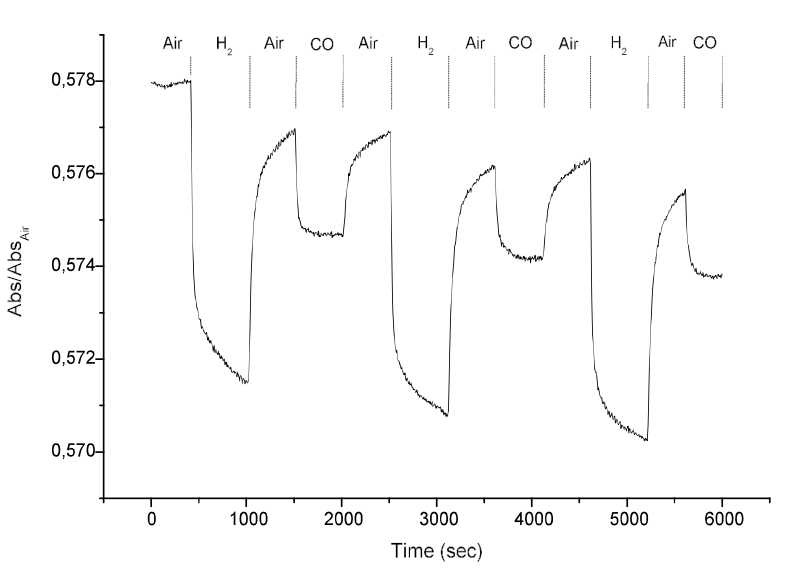

Figura 6. Ensayo de sensor gaseoso en función del tiempo para un compuesto de 75NiO-25ZnO-3\%Au a $380 \mathrm{~nm}$.

En la figura 6, se muestra el tiempo de respuesta del semiconductor ensayado a un mínimo OAC de $380 \mathrm{~nm}$, se puede notar que el compuesto $\mathrm{NiO}-\mathrm{ZnO}$ muestra una señal óptica detectable con la presencia de $\mathrm{Au}$, confirmando la respuesta óptica del $\mathrm{NiO}$ a los gases reductores. Esta muestra presenta una alta sensibilidad al gas hidrogeno y una baja respuesta al $\mathrm{CO}$, pero con una mejor respuesta respecto a las bajas temperatura ensayadas. Esto se puede deber a que los procesos cinéticos tales como adsorción son térmicamente activados, mejorando el rendimiento a altas temperaturas.

\section{CONCLUSIONES}

En resumen, se desarrollo un método simple y fácil para sintetizar un compuesto semiconductor con cristales homogéneos de $\mathrm{NiO}, \mathrm{ZnO}$ y $\mathrm{Au}$. El compuesto $75 \mathrm{NiO}-25 \mathrm{ZnO}$ dopado con $3 \%$ de nanopartículas de Au se ha caracterizado como un sensor óptico gaseoso para el $\mathrm{H}_{2}$. La caracterización funcional ha demostrado que las capas son sensibles ópticamente a los gases ensayados $\mathrm{CO}$ y $\mathrm{H}_{2}$, mostrando una excelente y rápida respuesta al gas $\mathrm{H}_{2}$, pero no muy buena para el $\mathrm{CO}$. La porosidad residual de la matriz del compuesto proporciona un camino para que las moléculas gaseosas lleguen hasta las nanopartículas finas incrustadas en el compuesto. Una simple comparación entre láminas dopados con nanopartículas de $\mathrm{Au}$ y láminas puras de $\mathrm{NiO}$ o $\mathrm{ZnO}$ permiten demostrar el efecto de mejoramiento a la sensibilidad de las nanopartículas de $A u$ en el desempeño de estos materiales.

\section{AGRADECIMIENTOS}

El presente trabajo es financiado por la Universidad de Padova a través del programa de intercambio académico BAPE ErasmusMundus.

\section{REFERENCIAS}

[1] R. Cavicchi, J. Suehle, P. Chaparala, K. Kreider, M. Gaitan, S. Semancik, Proceedings of the 1994 Solid State Sensor and Actuator Workshop, Hilton Head. (1994) 53-56.

[2] K.D. Schierbaum, S. Vaihinger, W. Gopel, H.H. Vlekkert, N.F. Rooij, Sensors and Actuators B, 1 (1990) 171-175.

[3] M.A. Butler, Sensors and Actuators B. 22 (1994) 155.

[4] C. Nylander, B. Liedberg, T. Lind, Sensors and Actuators. 3 (1982) 7988.

[5] A. Barkerm, S. Crowther, D. Ress, Surface room temperatura RF magnetrón sputtered $\mathrm{ZnO}$ for electromechanical devices, Sensors Actuators. A58 (3) (1997) 229.

[6] B. Wacogne, M, Roe, A. Pattinson, et al, Effective piezoelectric activity of zinc oxide films grown by radio frequency planar magnetron sputtering, Applied Physics Letters. 67 (12) (1995) 1674.

[7] L. Stolt, J. Hesdtrom, J. Kessler, et al., $\mathrm{ZnO} /$ CdS/ CuSnlnSe2 thin film solar cells with improved performance, Applied Physics Letters. 62 (3) (1993) 597.

[8] N. Zayer, R. Greerf, K. Rogers, et al., In situ monitoring of sputtered zinc oxide films for piezoelectric transducers, Thin Solid Films. 352 (2) (1999) 179.

[9] B. Ataev, A. Bagamadova, A. Djabrailov, et al., Highly conductive and transparent $\mathrm{Ga}$ doped epitaxial ZnO films on sapphire by CVD, Thin Solid Films. 260 (1) (1995) 19.

[10] B. Josph, K. Gopchandran, P. Thomas, et al., A study on the chemical spray deposition of zinc oxide thin films and their structural and electrical properties, Material Chemistry and Physics. 58 (1) (1999) 71.

[11] Z. Liu, Z. Jin, W. Li, et al., preparation of $\mathrm{ZnO}$ pororus thin films by sol-gel method using PEG template, Material Letters. 59 (2005) 3620-3625.

[12] D. Balk, S, Cho, Application of sol-gel derived films for $\mathrm{ZnO} / \mathrm{n}-\mathrm{Si}$ junction solar cells, Thin Solid Films. 354 (1999) 227.

[13] D. Bao, A. Kuang, H. Gu, Sol-gel derived caxis oriented $\mathrm{ZnO}$ thin films, Thin Solid Films. 312 (1998) 37.

[14] S. Berchams, H. Gomathi, G. Rao, J. Electroanal. Chem. 394 (1995) 267. 
[15] Z. Jiao, M. Wu, Z. Qin, $H . \quad X u$, Nanotechnology. 458 (2003) 14 (2003) 58.

[16] C. Makkus, K. Hemmes, D. Wir, J. Electroanal. Chem. 141 (1994) 3429.

[17] H. Guam, C. Shao, S. Ben, et al., Inorganic Chemical Commun. 6 (2003) 1302.

[18] E. Beach, K. Shqau, S. Brown, Material Chemical and Physics. 115 (2009) 371.

[19] M. Alagari, S. Ponnusamy, C. Muthamizchelvan, Syntehsis and characterization of $\mathrm{NiO}$ nanoparticles by solgel method, Mater Sci: Mater Electron. 6 (2011) 479

[20] D.R. Lide, Handbook of Chemistry and Physics, CRC press, 72nd Edition. (1991) 4112.

[21] R.J. Powell, W.E. Spicer, Phys. Rev. B. 2 (1970) 2182.

E-mail: didialfanet@hotmail.com 